\title{
Antibiotic Resistant Bacteria from Treated and Untreated Hospital Wastewater at Ayder Referral Hospital, Mekelle, North Ethiopia
}

\author{
Tsegahun Asfaw ${ }^{1,2}$, Letemichael Negash², Amlsha Kahsay², Yemane Weldu² \\ ${ }^{1}$ Department of Medical Laboratory Science, Medical Microbiology Unit, DebreBerhan University, Debre Berhan, Ethiopia \\ ${ }^{2}$ Department of Medical Microbiology and Immunology, Institute of Biomedical Sciences, Mekelle University, Mekelle, Ethiopia \\ Email: tsegahun.asfaw12@gmail.com
}

How to cite this paper: Asfaw, T., Negash, L., Kahsay, A. and Weldu, Y. (2017) Antibiotic Resistant Bacteria from Treated and Untreated Hospital Wastewater at Ayder Referral Hospital, Mekelle, North Ethiopia. Advances in Microbiology, 7, 871-886. https://doi.org/10.4236/aim.2017.712067

Received: November 9, 2017

Accepted: December 24, 2017

Published: December 27, 2017

Copyright $\odot 2017$ by authors and Scientific Research Publishing Inc. This work is licensed under the Creative Commons Attribution International License (CC BY 4.0).

http://creativecommons.org/licenses/by/4.0/ (c) (i) Open Access

\begin{abstract}
The widespread emergence of antibiotic resistance among bacterial pathogens has become one of the most serious challenges in Ethiopia. This study determined the prevalence and drug resistance patterns of bacterial pathogens isolated from treated and untreated wastewater released from Ayder Referral Hospital in Northern Ethiopia. A cross sectional study design was conducted from September-December, 2015 in wastewater released from Ayder referral hospital. A total of 40 composite samples were aseptically collected, transported and processed for enumeration of indicator organisms, bacteriological identification and susceptibility testing following standard procedure. Data obtained were entered and analyzed using SPSS version 20. Mean heterotrophic plate count, total coliform count, fecal coliform count and $E$. coli count were found to be $1.6 \times 10^{6} \mathrm{CFU} / \mathrm{mL}, 2.2 \times 10^{6} \mathrm{CFU} / 100 \mathrm{~mL}, 2.0 \times 10^{5}$ $\mathrm{CFU} / 100 \mathrm{~mL}$ and $1.1 \times 10^{4} \mathrm{CFU} / 100 \mathrm{~mL}$ from treated wastewater respectively. Among the total samples 134 bacterial isolates were detected and [84 (62.7\%)] were from untreated wastewater and [50 (37.3\%)] were from treated wastewater. The most frequently isolated bacteria from untreated wastewater samples was Klebsiella spp [14 (16.7\%)] followed by $S$. aureus $[13(15.5 \%)]$ and $P$. aeruginosa $[12(14.3 \%)]$, similarly in treated wastewater samples Klebsiella spp $[10(20 \%)], P$. aeruginosa $[8(16 \%)]$ and $S$. aureus $[8(16 \%)]$ were frequently detected. The overall multi-drug resistance (MDR) in this study was [79/134 (79.1\%)]. MDR from untreated wastewater sample was [64/84 $(76.2 \%)]$ while from treated wastewater sample was [42/50 (84\%)] and shows significant difference with (COR: 1.64, 95\% CI: 1.15 - 3.29, P: 0.001). It is concluded that treated hospital wastewater contains large numbers of antibiotic
\end{abstract}


resistant bacteria. Therefore, there should be continuous monitoring and evaluation of the effluent quality of the ponds and chlorination of the final effluent should be developed.

\section{Keywords}

Indicator Organism, Bacterial Isolates, Drug Resistance, Treated Wastewater, Untreated Wastewater

\section{Introduction}

Wastewater is an ideal media for a wide range of microorganisms especially bacteria, viruses and protozoa, and used as a reservoir for resistant bacteria [1]. It carries the resistant bacteria introduced into the sewage system that come from human excretions, liquid waste discharged from domestic home, agricultural and commercial sectors, pharmaceutical and hospitals [2] [3].

The volume of antibiotics used in hospitals released into effluent results a selection pressure on bacteria. Therefore, wastewater from hospitals contains high numbers of resistant bacteria and antibiotic residues at concentrations able to inhibit the growth of susceptible bacteria [4]. Accordingly, hospital wastewater could increase the numbers of resistant bacteria in the recipient sewers by both mechanisms of introduction and selection pressure [5]. The release of resistant bacteria to the receiving environment can pose public health impact through, carrying transmissible gene, by acting as a vector or reservoir of resistant gene [6] [7]. The most common bacterial pathogens found in hospital wastewater are Salmonella, Shigella, S. aureus, P. aeruginosa, Vibrio, Clostridium, Yersinia, Campylobacter, Leptospira and groups of total coliforms consisting of Serratia, Enterobacter, Klebsiella, and Escherichia coli (E. coli) [8] [9]. Those bacterial pathogens are among of the most dangerous contaminants on human health, and then the effluents from hospital wastewaters are one of the most serious pollutants discharging to the environment [9] [10]. The multidrug resistance patterns seen in the bacterial isolates from hospital effluent samples include most of the antibiotics used presently for treating human infections. The worst fear apprehended is the transfer of such resistance to bacterial pathogens causing infections in the community. In that case most of the presently available antibiotics will be vain against the infectious organisms. The origin of such multidrug resistance bacterial strains appears to be the hospital environment due to selective pressure [11]. Dissemination of antimicrobial resistance bacteria in the environment is a major problem in developing countries, mainly due to improper antibiotic usage, ineffective infection control program and lack of better management of hospital wastewater. In Ethiopia, rapid urbanization and industrialization with improper environmental planning often lead to discharge of industrial and hospital sewage effluent directly to the environment also a problem [12] [13]. 


\section{Materials and Methods}

\subsection{Study Area}

Ayder referral hospital has wastewater stabilization pond as treatment system. The treatment plant consists of seven rectangular slanty ponds. The ponds are lined at the bottom by thick plastic to minimize seepage into ground water zones. The first pond (facultative pond) is the largest of all, having greater length and width from the other ponds and receives raw wastewater. It is primarily designed for BOD removal and has three zones in vertical section; those are supper aerobic zone, middle facultative zone or intermediate zone and lower anaerobic zone. Maturation ponds consist of six slanty ponds having almost similar length and width and have shallow depth. It receives wastewater effluent from facultative pond and its primary function is removal of pathogenic microorganisms. The last step in treatment process is release of treated effluent to open field continuously after flowing approximately 50 meter sewage system. The Untreated Hospital Wastewater (UHWW) sampling site was just the inlet of treatment plant while the Treated hospital Wastewater (THWW) sampling site was just at the outlet of treatment plant before released to the open field.

\subsection{Study Design and Period}

Cross sectional study design was conducted to collect hospital wastewater samples from September, 2015 to December, 2015.

\subsection{Sampling Frequency and Sampling Technique}

A "Composite-sampling" technique was applied to collect the most representative samples according to guidelines of wastewater sampling techniques stated on APHA [14]. A total of 80 partial samples were collected from both sampling site with 4 hour interval in the study period and finally 40 composite samples were processed for bacteriological analysis, identification and susceptibility testing. Partial samples were collected two times a day with four hour interval from both sites in $125 \mathrm{~mL}$ cleaned and sterile microbiological glass bottles. The first samples were collected in the morning $10 \mathrm{AM}$ from UHWW sampling site and 10:30 AM from THWW sampling site and transported immediately after collection and stored in refrigerator at $4^{\circ} \mathrm{C}$. After collection of afternoon samples 2 PM from UHWW and 2:30 PM from THWW, the partial samples were pooled (untreated to untreated and treated to treated) aseptically to $250 \mathrm{~mL}$ sized cleaned and sterilized microbiological glass bottle. Then it was stored in refrigerator at $4^{\circ} \mathrm{C}$ until the samples is processed.

\subsection{Bacteriological Analysis of Wastewater}

Total heterotrophic plate count: Duplicate plates were prepared for each volume of sample examined. All the samples were vigorously shaken before preparation of dilutions then serial 10-fold dilutions of samples were prepared in physiological saline, and $1 \mathrm{~mL}$ aliquot was spread over nutrient agar plate (Himedia). 
Then plates were incubated for $48 \mathrm{~h}$ at $37^{\circ} \mathrm{C}$ before bacteriological counts were performed. Number of colonies on duplicate plate having 30 - 300 colonies was counted and finally bacterial count was reported as $\mathrm{CFU} / \mathrm{mL}$.

Total coliform count: Serial 10-fold dilutions of sample were prepared in physiological saline and $1 \mathrm{~mL}$ of aliquot was transferred aseptically in to a series of 9 test tubes containing Durham tubes and double strength MacConkey broth (Himedia). Tubes were gently shaken and incubated for $24-48 \mathrm{~h}$ at $37^{\circ} \mathrm{C}$, then production of gas and lactose fermentation was taken as positive reaction. Finally bacterial load were estimated using MPN table and dilution factor. The bacterial count was reported as $\mathrm{CFU} / 100 \mathrm{~mL}$.

Fecal coliform count: Serial 10-fold dilutions of sample were prepared in physiological saline and $1 \mathrm{~mL}$ of aliquot was transferred aseptically in to a series of 9 test tubes containing Durham tubes and double strength MacConkey broth. Tubes were gently shaken and incubated for $24 \mathrm{~h}$ at $44.5^{\circ} \mathrm{C}$ in water bath, then production of gas and lactose fermentation was taken as positive reaction. Furthermore E. coli cont was confirmed by adding approximately $0.1 \mathrm{ml}$ of Kovacs reagent (Uni-Chem). Finally bacterial load were estimated using MPN table and dilution factor. The bacterial count was reported as CFU/100 mL.

\subsection{Isolation and Identification of Bacteria}

Culture technique: The sample was investigated for further isolation and identification of bacterial pathogens. Representative diluted sample of $1 \mathrm{ml}$ aliquots was plated on selective and differential media. MacConkey agar (SRL-sisco research laboratory), Salmonella-shigella agar (Uni-Chem) and Mannitol salt agar (Himedia) were used and prepared according to manufacturer direction. After obtaining pure colonies and recording important features of the isolated organisms, further identification were done using gram staining and biochemical test with standard methods.

Biochemical test: Gram negative bacteria was identified based on colonial morphology and pigmentation, Oxidase test, Carbohydrate fermentation, $\mathrm{H}_{2} \mathrm{~S}$ production, Citrate utilization, motility, growth at $42^{\circ} \mathrm{C}$, Indole formation, Lysine decarboxylase and Lysine deaminase production, and Urea hydrolysis. Gram positive isolates were also differentiated by gram staining, colonial characteristics, catalase test and coagulase tests.

\subsection{Antimicrobial Susceptibility Testing}

A standard Kirby-Bauer disk diffusion method was used to determine the antimicrobial susceptibility profiles of the isolates as described by the CLSI, 2014 [15]. Bacterial isolates were tested for the following commonly prescribed drug: Erythromycin $(15 \mu \mathrm{g})$, Gentamyacin $(10 \mu \mathrm{g})$, Amikacin $(30 \mu \mathrm{g})$, Amoxicillin-Clavulunic acid $(30 \mu \mathrm{g})$, Ceftriaxone $(30 \mu \mathrm{g})$, Ciprofloxacin $(5 \mu \mathrm{g})$, Tetracycline $(30 \mu \mathrm{g})$, Cotrimoxazole $(25 \mu \mathrm{g})$, Ampicillin $(10 \mu \mathrm{g})$, Penicillin $(10 \mu \mathrm{g})$, Chloromaphenicol $(30 \mu \mathrm{g})$, Doxycycline $(30 \mu \mathrm{g})$ and Cefoxitin $(30 \mu \mathrm{g})$. Interpretation was made using susceptibility breakpoints of CLSI, 2014 and diameter of 
the zone of inhibition around the disc was measured to the nearest millimeter using a metal caliper and the isolates were classified as sensitive, intermediate and resistant.

\subsection{Data Quality Assurance}

Sample collection, handling, transportation and microbiological analysis and interpretation of results were carried out using standard operating procedures (SOPs). Prior to the actual work Reagents, media and antimicrobial disks were checked for expiry date, damage and storage problems. Laboratory equipment's were properly cleaned and sterilized before use. Media preparation was made based on the respective manufacturer's directions. 5\% of media per batch/prepared was incubated overnight for sterility check. Escherichia coli (ATCC 25922), Staphylococcus aureus (ATCC 25923) and Pseudomonas aeruginosa (ATCC 28753) were used as quality control organisms.

\subsection{Data Management and Statistical Analysis}

Data was entered and summarized using SPSS version 20 software (USA) and analyzed using the STATA software (StataCorp LP, College. Station, Texas, USA). A $p$-value of $\leq 0.05$ was considered indicative of a statistically significant difference.

\section{Results}

\subsection{Bacteriological Analysis of Wastewater before and after Treatment}

Mean heterotrophic plate count, total coliform count, fecal coliform count and E. coli count were found to be $1.6 \times 10^{6} \mathrm{CFU} / \mathrm{mL}, 2.2 \times 10^{6} \mathrm{CFU} / 100 \mathrm{~mL}, 2.0 \times$ $10^{5} \mathrm{CFU} / 100 \mathrm{~mL}$ and $1.1 \times 10^{4} \mathrm{CFU} / 100 \mathrm{~mL}$ from THWW respectively (Table 1 ).

\subsection{Bacterial Isolates}

Among the total samples 134 bacterial isolates were detected and $84(62.7 \%)$ were from UHWW and 50 (37.3\%) were from THWW. The most frequently isolated bacteria from untreated wastewater samples was Klebsiella spp 14 (16.7\%) followed by S. aureus 13 (15.5\%) and P. aeruginosa 12 (14.3\%), similarly in treated wastewater samples Klebsiella spp 10 (20\%), P. aeruginosa 8 (16\%) and $S$. aureus 8 (16\%) were frequently isolated (Table 2 ).

Table 1. Mean of indicator organisms in treated and untreated wastewater released from Ayder referral hospital, Mekelle, North Ethiopia (September-December 2015).

\begin{tabular}{ccc}
\hline Indicator organism & UHWW $(\mathbf{n}=\mathbf{2 0})$ & THWW $(\mathbf{n}=\mathbf{2 0})$ \\
\hline Total heterotrophic count $(\mathrm{CFU} / \mathrm{mL})$ & $1.9 \times 10^{8}$ & $1.6 \times 10^{6}$ \\
Total coliform count $(\mathrm{CFU} / 100 \mathrm{~mL})$ & $2.6 \times 10^{10}$ & $2.2 \times 10^{6}$ \\
Fecal coliform count $(\mathrm{CFU} / 100 \mathrm{~mL})$ & $1.25 \times 10^{9}$ & $2.0 \times 10^{5}$ \\
E. coli count $(\mathrm{CFU} / 100 \mathrm{~mL})$ & $4.5 \times 10^{5}$ & $1.1 \times 10^{4}$ \\
\hline
\end{tabular}


Table 2. Frequency of bacterial isolates from treated and untreated wastewater released from Ayder referral hospital, Mekelle, North Ethiopia (September-December 2015).

\begin{tabular}{cccc}
\hline Bacteria isolates & UHWW $(\mathbf{n}=\mathbf{2 0})$ & THWW $(\mathbf{n}=20)$ & Total $(\mathbf{n}=40)$ \\
\hline S. aureus & $13(15.5 \%)$ & $8(16 \%)$ & $21(15.7 \%)$ \\
CoNS & $6(7.1 \%)$ & $5(10 \%)$ & $11(8.2 \%)$ \\
Klebsiella spp & $14(16.7 \%)$ & $10(20 \%)$ & $24(17.9 \%)$ \\
P. aeruginosa & $12(14.3 \%)$ & $8(16 \%)$ & $20(14.9 \%)$ \\
E. coli & $11(13.1 \%)$ & $6(12 \%)$ & $17(12.7 \%)$ \\
Salmonella spp & $9(10.7)$ & $6(12 \%)$ & $15(11.2 \%)$ \\
Shigella spp & $3(3.6 \%)$ & $0(0)$ & $3(2.2 \%$ \\
Citrobacter spp & $6(7.1 \%)$ & $3(6 \%)$ & $9(6.7 \%)$ \\
Enterobacter spp & $4(4.8 \%)$ & $2(4 \%)$ & $6(4.5 \%)$ \\
Other isolates & $6(7.1 \%)$ & $2(4 \%)$ & $8(6 \%)$ \\
Total & $\mathbf{8 4}(100 \%)$ & $50(100 \%)$ & $134(100 \%)$ \\
\hline
\end{tabular}

${ }^{*}$ CoNS: Coagulase negative staphylococci, other isolates: Acinitobacter spp (only 1 in UHWW), Seratia spp ( 3 in UHWW and 1 in THWW) and Proteus spp ( 2 in UHWW and 1 in THWW).

\subsection{Drug Susceptibility Profile of Bacterial Isolates}

Among bacterial isolates from untreated wastewater, Coagulase negative staphylococci (CoNS) were found to be $100 \%$ resistant to Penicillin and $50 \%$ to Cefoxitin. S. aureus was also found to be $77 \%$ and $38 \%$ resistant for Penicillin and Cefoxitin respectively. All isolates of E. coli and Klebsiella spp were 100\% resistant to ampicillin (Table 3).

The total resistance of bacterial isolates from untreated wastewater was higher for penicillin 16/19 (84\%) followed by ampicillin 42/52 (81\%) and tetracycline 30/72 (42\%). However, relatively lower resistance was observed among bacterial isolates to Chloramphenicol 13/71 (18\%), gentamycin 19/84 (23\%) and ciprofloxacin 20/84 (24\%) (Figure 1).

Among bacterial isolates from treated wastewater, all isolates of $S$. aureus and CoNS were $100 \%$ resistant to penicillin. All isolates of E. coli, Klebsiella spp and Citrobacter spp were $100 \%$ resistant to ampicillin as shown in the (Table 4).

The total resistance of isolates from treated wastewater to penicillin was 13/13 (100\%) followed by ampicillin 24/29 (83\%) and Ceftriaxone 17/29 (59\%). Relatively lower resistance among bacterial isolates was observed to ciprofloxacin 10/50 (20\%), amikacin 10/50 (20\%) and chloramphenicol 10/42 (24\%) (Figure 2).

Among isolates from untreated wastewater, 19/72 (26.3\%) were resistant for six and more antibiotics (e.g. three isolates of klebsiella spp and one isolates of $E$. coli were resistant to nine antibiotics; two isolates of klebsiella spp, two isolates of E. coli and one isolates of Citrobacter spp were resistant to eight antibiotics), while $13 / 84(15.5 \%)$ isolates were resistant for only one antibiotic, and 7/84 (8.3\%) were not resistant for any of antibiotics tested (Table 5). 
Table 3. Drug Susceptibility profile of bacterial isolates from untreated wastewater released from Ayder referral hospital, Mekelle, North Ethiopia (September-December 2015).

\begin{tabular}{|c|c|c|c|c|c|c|c|c|c|c|c|c|c|c|}
\hline \multirow{2}{*}{ Bacteria isolates } & & \multicolumn{13}{|c|}{ Antibiotics used N (\%) } \\
\hline & & GN & AK & TTC & DO & SXT & CIP & $\mathrm{CAF}$ & AMP & CRO & AMC & $\mathrm{E}$ & $\mathbf{P}$ & $\mathrm{CX}$ \\
\hline \multirow{3}{*}{ S. aureus (13) } & $\mathbf{R}$ & $2(15)$ & $4(31)$ & $3(23)$ & $1(8)$ & $2(15)$ & $2(15)$ & $4(31)$ & NT & NT & NT & $3(23)$ & $10(77)$ & $5(38)$ \\
\hline & I & $3(23)$ & $3(23)$ & $4(31)$ & $6(46)$ & $3(23)$ & $2(15)$ & $0(0)$ & NT & NT & NT & $1(8)$ & - & - \\
\hline & $\mathrm{S}$ & $8(62)$ & $6(46)$ & $6(46)$ & $6(46)$ & $8(62)$ & $9(69)$ & $9(69)$ & NT & NT & NT & $9(69)$ & $3(23)$ & $8(62)$ \\
\hline \multirow{3}{*}{$\operatorname{CoNS}(6)$} & $\mathbf{R}$ & $0(0)$ & $1(17)$ & $3(50)$ & $2(33)$ & $2(23)$ & $1(17)$ & $1(17)$ & NT & NT & NT & $2(33)$ & $6(100)$ & $3(50)$ \\
\hline & I & $3(50)$ & $3(50)$ & $2(23)$ & $0(0)$ & $1(17)$ & $2(23)$ & $2(23)$ & NT & NT & NT & $2(33)$ & - & - \\
\hline & $\mathrm{S}$ & $3(50)$ & $2(33)$ & $1(17)$ & $4(66)$ & $3(50)$ & $3(50)$ & $3(50)$ & NT & NT & NT & $2(33)$ & $0(0)$ & $3(50)$ \\
\hline \multirow{3}{*}{ Klebsiella spp (14) } & $\mathbf{R}$ & $6(43)$ & $6(43)$ & $9(64)$ & $7(50)$ & $6(43)$ & $4(29)$ & $2(14)$ & $14(100)$ & $6(43)$ & $5(36)$ & NT & NT & NT \\
\hline & I & $2(14)$ & $3(21)$ & $1(7)$ & $3(21)$ & $2(14)$ & $3(21)$ & $4(29)$ & $0(0)$ & $3(21)$ & $2(14)$ & NT & NT & NT \\
\hline & $S$ & $6(43)$ & $5(36)$ & $4(29)$ & $4(29)$ & $6(43)$ & $7(50)$ & $8(57)$ & $0(0)$ & $5(36)$ & $7(50)$ & NT & NT & NT \\
\hline \multirow{3}{*}{ E. coli $(11)$} & $\mathbf{R}$ & $2(18)$ & $2(18)$ & $4(36)$ & $2(18)$ & $5(45)$ & $3(27)$ & $1(9)$ & $11(100)$ & $4(36)$ & $3(27)$ & NT & NT & NT \\
\hline & I & $3(27)$ & $2(18)$ & $3(27)$ & $3(27)$ & $2(18)$ & $3(27)$ & $2(18)$ & $0(0)$ & $2(18)$ & $2(18)$ & NT & NT & NT \\
\hline & $S$ & $6(55)$ & $7(64)$ & $4(36)$ & $6(55)$ & $4(36)$ & $5(45)$ & $8(73)$ & $0(0)$ & $5(45)$ & $6(55)$ & NT & NT & NT \\
\hline \multirow{3}{*}{ P. aeruginosa (12) } & $\mathbf{R}$ & $4(33)$ & $4(33)$ & NT & NT & NT & $3(25)$ & NT & NT & NT & NT & NT & NT & NT \\
\hline & I & $2(17)$ & $2(17)$ & NT & NT & NT & $4(33)$ & NT & NT & NT & NT & NT & NT & NT \\
\hline & $\mathrm{S}$ & $6(50)$ & $6(50)$ & NT & NT & NT & $5(42)$ & NT & NT & NT & NT & NT & NT & NT \\
\hline \multirow{3}{*}{ Salmonella spp (9) } & $\mathbf{R}$ & $0(0)$ & $1(11)$ & $3(33)$ & $2(22)$ & $1(11)$ & $2(22)$ & $1(11)$ & $5(56($ & $3(33)$ & $3(33)$ & NT & NT & NT \\
\hline & I & $4(44)$ & $2(22)$ & $2(22)$ & $2(22)$ & $3(33)$ & $2(22)$ & $3(33)$ & $2(22)$ & $2(22)$ & $0(0)$ & NT & NT & NT \\
\hline & $\mathrm{S}$ & $5(56)$ & $6(67)$ & $4(44)$ & $5(56)$ & $5(56)$ & $5(56)$ & $5(56)$ & $2(22)$ & $4(44)$ & $6(67)$ & NT & NT & NT \\
\hline \multirow{3}{*}{ Shigella spp (3) } & $\mathbf{R}$ & $1(33$ & $0(0)$ & $0(0)$ & $0(0)$ & $1(33)$ & $1(33)$ & $1(33)$ & $2(67)$ & $2(67)$ & $1(33)$ & NT & NT & NT \\
\hline & I & $0(0)$ & $1(33)$ & $1(33)$ & $1(33)$ & $1(33)$ & $0(0)$ & $0(0)$ & $0(0)$ & 0 & $0(0)$ & NT & NT & NT \\
\hline & $\mathrm{S}$ & $2(67)$ & $2(67)$ & $2(67)$ & $2(67)$ & $1(33)$ & $2(67)$ & $2(67)$ & $1(33)$ & $1(33)$ & $2(67)$ & NT & NT & NT \\
\hline \multirow{3}{*}{ Citrobacter spp (6) } & $\mathbf{R}$ & $2(33)$ & $2(33)$ & $3(50)$ & $2(33)$ & $2(33)$ & $2(33)$ & $1(17)$ & $5(83)$ & $3(50)$ & $1(17)$ & NT & NT & NT \\
\hline & I & $1(17)$ & $0(0)$ & $1(17)$ & $1(17)$ & $1(17)$ & $0(0)$ & $2(33)$ & $1(17)$ & $1(17)$ & $3(50)$ & NT & NT & NT \\
\hline & $\mathrm{S}$ & $3(50)$ & $4(67)$ & $2(33)$ & $3(50)$ & $3(50)$ & $4(67)$ & $3(50)$ & $0(0)$ & $2(33)$ & $2(33)$ & NT & NT & NT \\
\hline \multirow{3}{*}{ Enterobacter spp (4) } & $\mathbf{R}$ & $1(25)$ & $1(25)$ & $2(50)$ & $1(25$ & $1(25)$ & $1(25)$ & $1(25)$ & $2(50)$ & $0(0)$ & $1(25)$ & NT & NT & NT \\
\hline & I & $2(50)$ & $3(75)$ & $0(0)$ & $2(50)$ & $3(75)$ & $3(75)$ & $0(0)$ & $1(25)$ & $3(75)$ & $2(50)$ & NT & NT & NT \\
\hline & $\mathrm{s}$ & $1(25)$ & $0(0)$ & $2(50)$ & $1(25)$ & $0(0)$ & $0(0)$ & $3(75)$ & $1(25)$ & $1(25)$ & $1(25)$ & NT & NT & NT \\
\hline \multirow{3}{*}{ Acinetobacter spp (1) } & $\mathbf{R}$ & $0(0)$ & $0(0)$ & $1(100)$ & $1(100)$ & $1(100)$ & $0(0)$ & NT & NT & $1(100)$ & NT & NT & NT & NT \\
\hline & I & $1(100)$ & $0(0)$ & $0(0)$ & $0(0)$ & $0(0)$ & $1(100)$ & NT & NT & $0(0)$ & NT & NT & NT & NT \\
\hline & $S$ & $0(0)$ & $1(100$ & $0(0)$ & $0(0)$ & $0(0)$ & $0(0)$ & NT & NT & $0(0)$ & NT & NT & NT & NT \\
\hline \multirow{3}{*}{ Other isolates (5) } & $\mathbf{R}$ & $1(20)$ & $1(20)$ & $2(40)$ & $1(20)$ & $1(20)$ & $1(20)$ & $1(20)$ & $3(60)$ & $2(40)$ & $2(40)$ & NT & NT & NT \\
\hline & I & $2(40)$ & $3(60)$ & $1(20)$ & $2(40)$ & $3(60)$ & $3(60)$ & $3(60)$ & $1(20)$ & $0(0)$ & $1(20)$ & NT & NT & NT \\
\hline & $S$ & $2(40)$ & $1(20)$ & $2(20)$ & $2(40)$ & $1(20)$ & $1(20)$ & $1(20)$ & $1(20)$ & $3(60)$ & $2(40)$ & NT & NT & NT \\
\hline
\end{tabular}

GN: Gentamycin, TTC: Tetracyclin, SXT: Cotrimoxazole, CAF: Chloramphenicol, P: Penicillin, AK: Amikacin, DO: Doxycycline, CIP: Ciprofloxacin, E: Erytromyacin, CX: Cefoxitin, AMC: Amoxacillin-clavulunic acid, AMP: Ampicillin, CRO: Ceftriaxone, R: Resistance, S: Susceptible, I, Intermediate, NT: Not Tested, Other isolates: Seratia spp (3) and Proteus spp (2). 
Table 4. Drug Susceptibility profile of bacterial isolates from treated wastewater released from Ayder referral hospital, Mekelle, North Ethiopia (September-December 2015).

\begin{tabular}{|c|c|c|c|c|c|c|c|c|c|c|c|c|c|c|}
\hline \multirow{2}{*}{ Bacteria isolates } & & \multicolumn{13}{|c|}{ Antibiotics used N (\%) } \\
\hline & & GN & $\mathrm{AK}$ & TTC & DO & SXT & CIP & CAF & AMP & CRO & AMC & E & $\mathbf{P}$ & $\mathrm{CX}$ \\
\hline \multirow{3}{*}{ S. aureus (8) } & $\mathbf{R}$ & $2(25)$ & $1(13)$ & $2(25)$ & $3(38)$ & $1(13)$ & $1(13)$ & $2(25)$ & NT & NT & NT & $3(38)$ & $8(100)$ & $3(38)$ \\
\hline & I & $1(13)$ & $4(50)$ & $3(38)$ & $2(25)$ & $4(50)$ & $3(38)$ & $1(13)$ & NT & NT & NT & $1(13)$ & - & - \\
\hline & $\mathrm{S}$ & $5(63)$ & $3(38)$ & $3(38)$ & $3(38)$ & $3(38)$ & $4(50)$ & $5(63)$ & NT & NT & NT & $4(50)$ & $0(0)$ & $5(63)$ \\
\hline \multirow{3}{*}{ CoNS (5) } & $\mathbf{R}$ & $1(20)$ & $0(0)$ & $2(40)$ & $2(40)$ & $1(20)$ & $0(0)$ & $2(40)$ & NT & NT & NT & $1(20)$ & $5(100)$ & $2(40)$ \\
\hline & I & $3(60)$ & $2(40)$ & $0(0)$ & $0(0)$ & $0(0)$ & $2(40)$ & $0(0)$ & NT & NT & NT & $3(60)$ & - & - \\
\hline & $S$ & $1(20)$ & $3(60)$ & $3(60)$ & $3(60)$ & $4(80)$ & $3(60)$ & $3(60)$ & NT & NT & NT & $1(20)$ & $0(0)$ & $3(60)$ \\
\hline \multirow{3}{*}{ Klebsiella spp (10) } & $\mathbf{R}$ & $6(60)$ & $4(40)$ & $4(40)$ & $4(40)$ & $6(60)$ & $2(20)$ & $2(20)$ & $10(100)$ & $5(50)$ & $3(30)$ & NT & NT & NT \\
\hline & I & $0(0)$ & $1(10)$ & $2(20)$ & $3(30)$ & $0(0)$ & $4(40)$ & $3(30)$ & $0(0)$ & $1(10)$ & $3(30)$ & NT & NT & NT \\
\hline & $S$ & $4(40)$ & $5(50)$ & $4(40)$ & $3(30)$ & $4(40)$ & $4(40)$ & $5(50)$ & $0(0)$ & $4(40)$ & $4(40)$ & NT & NT & NT \\
\hline \multirow{3}{*}{ E. coli $(6)$} & $\mathbf{R}$ & $2(33)$ & $2(33)$ & $2(33)$ & $2(33)$ & $4(67)$ & $2(33)$ & $1(17)$ & $6(100)$ & $3(50)$ & $3(50$ & NT & NT & NT \\
\hline & I & $1(17)$ & $0(0)$ & $0(0)$ & $1(17)$ & $0(0)$ & $1(17)$ & $1(17)$ & $0(0)$ & $2(33)$ & $1(17)$ & NT & NT & NT \\
\hline & $S$ & $3(50)$ & $4(67)$ & $4(67)$ & $3(50)$ & $2(33)$ & $3(50)$ & $4(67)$ & $0(0)$ & $1(17)$ & $2(33)$ & NT & NT & NT \\
\hline \multirow{3}{*}{ P. aeruginosa (8) } & $\mathbf{R}$ & $4(50)$ & $2(25)$ & NT & NT & NT & $3(38)$ & NT & NT & NT & NT & NT & NT & NT \\
\hline & I & $2(25)$ & $4(50)$ & NT & NT & NT & $0(0)$ & NT & NT & NT & NT & NT & NT & NT \\
\hline & $S$ & $2(25)$ & $2(25)$ & NT & NT & NT & $5(62)$ & NT & NT & NT & NT & NT & NT & NT \\
\hline \multirow{3}{*}{ Salmonella spp (6) } & $\mathbf{R}$ & $2(33)$ & $0(0)$ & $3(50)$ & $2(33)$ & $2(33)$ & $1(17)$ & $1(17)$ & $3(50)$ & $4(67)$ & $2(33)$ & NT & NT & NT \\
\hline & I & $1(17)$ & $2(33)$ & $0(0)$ & $0(0)$ & $1(17)$ & $2(33)$ & $2(33)$ & $0(0)$ & $0(0)$ & $1(17)$ & NT & NT & NT \\
\hline & $S$ & $3(50)$ & $4(67)$ & $3(50)$ & $4(67)$ & $3(50)$ & $3(50)$ & $3(50)$ & $3(50)$ & $2(33)$ & $3(50)$ & NT & NT & NT \\
\hline \multirow{3}{*}{ Citrobacter spp (3) } & $\mathbf{R}$ & $1(33)$ & $1(33)$ & $3(100)$ & $2(67)$ & $1(33)$ & $1(33)$ & $1(33)$ & $3(100)$ & $2(67)$ & $2(67)$ & NT & NT & NT \\
\hline & I & $2(67)$ & $0(0)$ & $0(0)$ & $0(0)$ & $2(67)$ & $0(0)$ & 0 & $0(0)$ & $1(33)$ & $0(0)$ & NT & NT & NT \\
\hline & $S$ & $0(0)$ & $2(67)$ & $0(0)$ & $1(33)$ & $0(0)$ & $2(67)$ & $2(67)$ & $0(0)$ & $0(0)$ & $1(33)$ & NT & NT & NT \\
\hline \multirow{3}{*}{ Enterobacter spp (2) } & $\mathbf{R}$ & $0(0)$ & $0(0)$ & $1(50)$ & $1(50)$ & $1(50)$ & $0(0)$ & 0 & $1(50)$ & $1(50)$ & $1(50$ & NT & NT & NT \\
\hline & I & $2(100)$ & $2(100)$ & $1(50)$ & $0(0)$ & $0(0)$ & $2(100)$ & $1(50)$ & $1(50)$ & $0(0)$ & $1(50)$ & NT & NT & NT \\
\hline & $S$ & $0(0)$ & $0(0)$ & $0(0)$ & $1(50)$ & $1(50)$ & $0(0)$ & $1(50)$ & $0(0)$ & $1(50)$ & $0(0)$ & NT & NT & NT \\
\hline \multirow{3}{*}{ Other isolates (2) } & $\mathbf{R}$ & $1(50)$ & $0(0)$ & $2(100)$ & $1(50)$ & $1(50)$ & $0(0)$ & $1(50)$ & $1(50)$ & $2(100)$ & $2(100)$ & NT & NT & NT \\
\hline & I & $1(50)$ & $1(50)$ & $0(0)$ & $0(0)$ & $1(50)$ & $2(100)$ & 0 & $1(50)$ & $0(0)$ & $0(0)$ & NT & NT & NT \\
\hline & $S$ & $0(0)$ & $1(50)$ & $0(0)$ & $1(50)$ & $0(0)$ & $0(0)$ & $1(50)$ & $0(0)$ & $0(0)$ & $0(0)$ & NT & NT & NT \\
\hline
\end{tabular}

GN: Gentamycin, TTC: Tetracyclin, SXT: Cotrimoxazole, CAF: Chloramphenicol, P: Penicillin, AK: Amikacin, DO: Doxycycline, CIP: Ciprofloxacin, E: Erytromyacin, CX: Cefoxitin, AMC: Amoxacillin-clavulunic acid, AMP: Ampicillin, CRO: Ceftriaxone R: Resistance, S: Susceptible, I, Intermediate, NT: Not Tested, Other isolates: Seratia spp (1) and Proteus spp (1). 


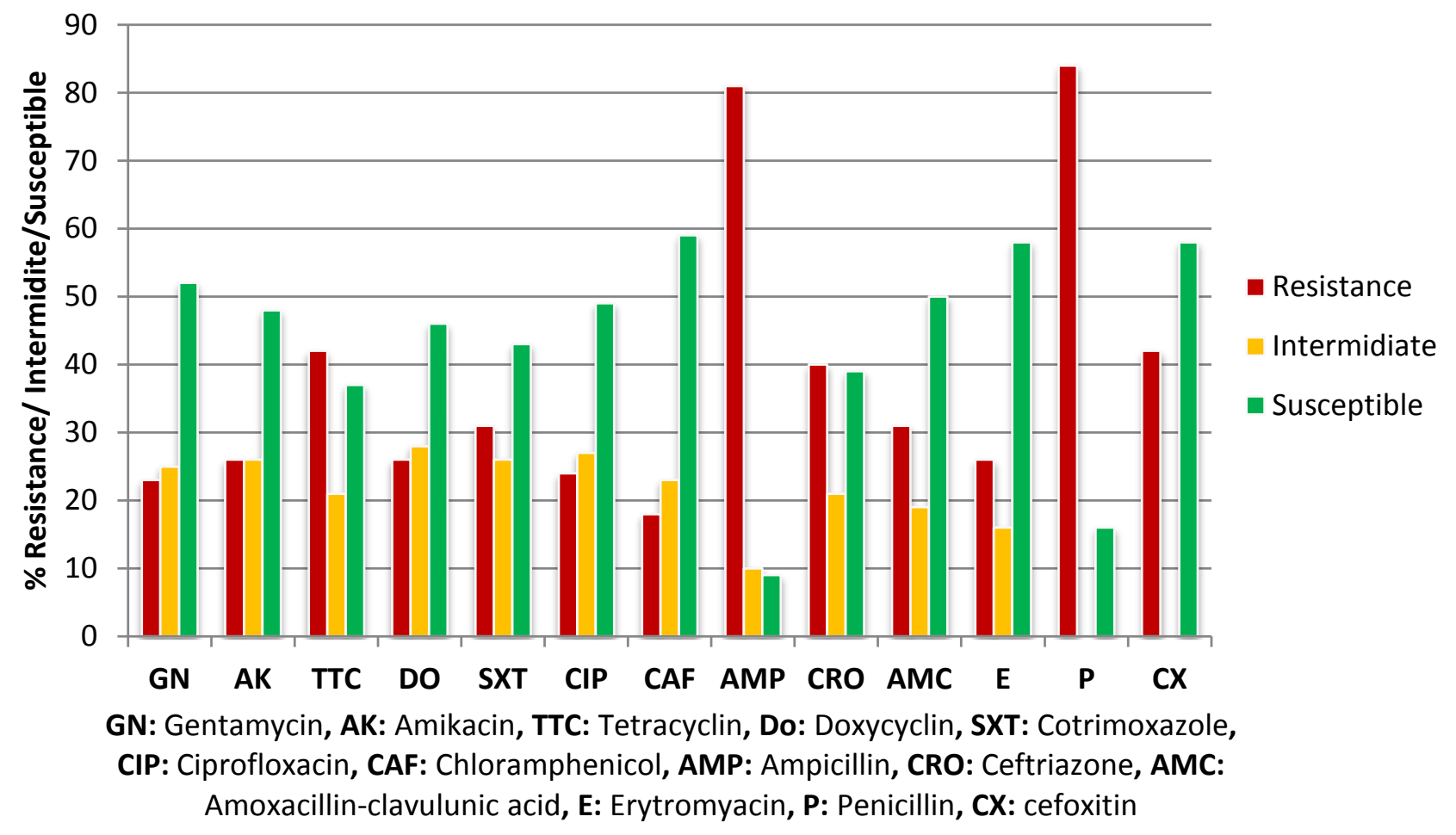

Figure 1. Total drug susceptibility profile of isolates from untreated wastewater released from Ayder referral hospital, Mekelle, North Ethiopia (September-December 2015).

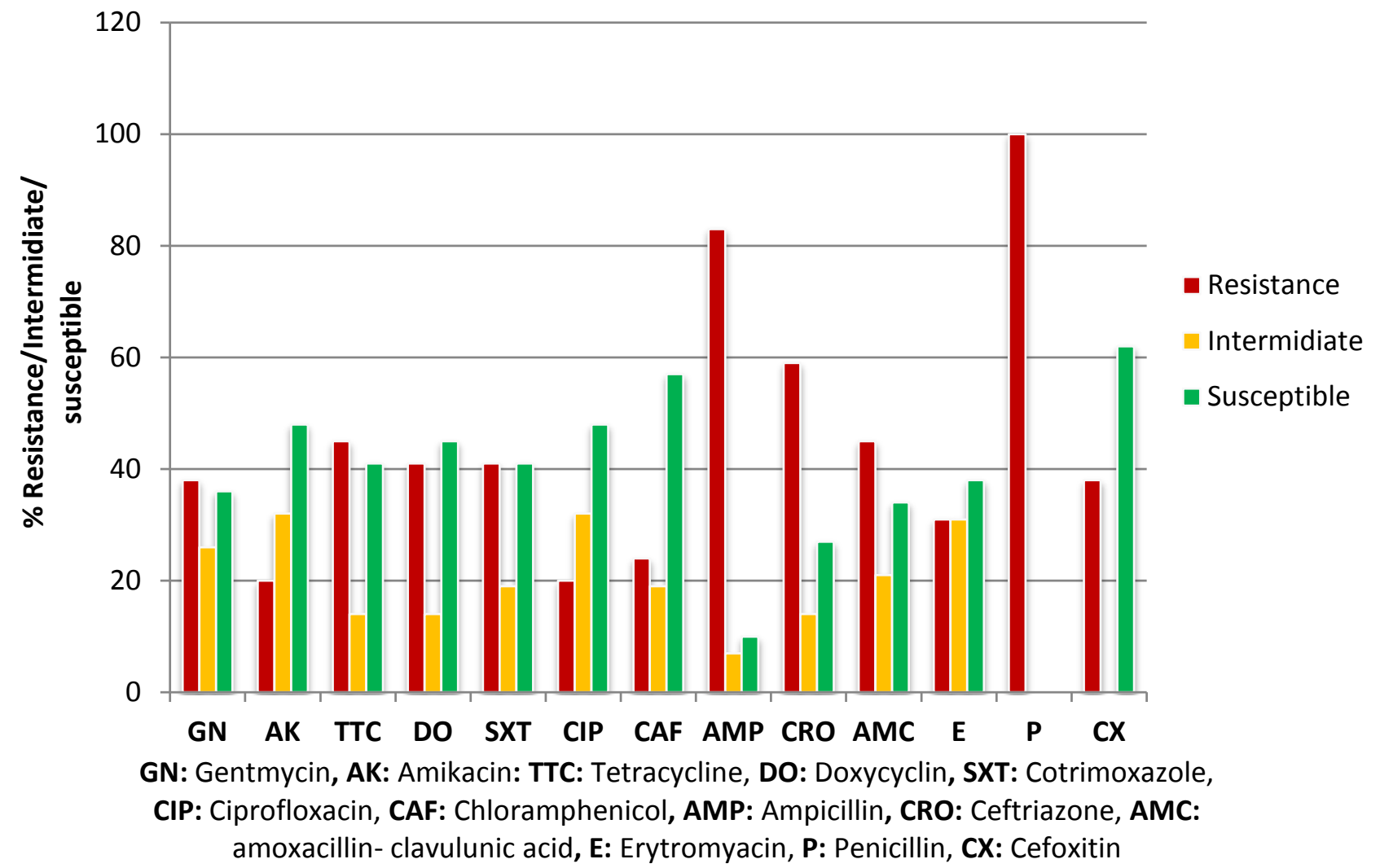

Figure 2. Total drug susceptibility profile of isolates from treated wastewater released from Ayder referral hospital, Mekelle, North Ethiopia (September-December 2015). 
Table 5. Multi-drug resistance patterns of bacterial isolates from untreated wastewater released from Ayder Referral Hospital, Mekelle, North Ethiopia (September-December 2015).

\begin{tabular}{ccccccccc}
\hline Bacteria isolates & $\begin{array}{c}\text { Number } \\
\text { of isolates }\end{array}$ & R0 & R1 & R2 & R3 & R4 & R5 & $\geq$ R6 \\
\hline $\begin{array}{c}\text { S. aureus } \\
\text { CoNS }\end{array}$ & 13 & $0(0)$ & $3(23.1)$ & $4(30.8)$ & $3(23.1)$ & $0(0)$ & $1(7.7)$ & $2(15.4)$ \\
Klebsiella spp & 14 & $0(0)$ & $1(7.1)$ & $3(21.4)$ & $1(7.1)$ & $0(0)$ & $1(7.1)$ & $8(57.1)$ \\
$\quad$ E. coli & 11 & $0(0)$ & $2(18.2)$ & $2(18.2)$ & $1(9.1)$ & $1(9.1)$ & $2(18.2)$ & $3(27.3)$ \\
P. aeroginosa & 12 & $5(41.7)$ & $2(18.2)$ & $3(25)$ & $2(18.2)$ & $\mathrm{NT}$ & $\mathrm{NT}$ & $\mathrm{NT}$ \\
Salmonella spp & 9 & $1(11.1)$ & $0(0)$ & $2(22.2)$ & $1(11.1)$ & $1(11.1)$ & $2(22.2)$ & $2(22.2)$ \\
Shigella spp & 3 & $1(33.3)$ & $1(33.3)$ & $0(0)$ & $0(0)$ & $0(0)$ & $0(0)$ & $1(33.3)$ \\
Citrobacter $\mathrm{spp}$ & 6 & $0(0)$ & $2(33.3)$ & $0(0)$ & $0(0)$ & $0(0)$ & $2(33.3)$ & $2(33.3)$ \\
Entrobacter spp & 4 & $0(0)$ & $2(50.0)$ & $1(25)$ & $0(0)$ & $1(25)$ & $0(0)$ & $0(0)$ \\
Other isolates & 6 & $0(0)$ & $0(0)$ & $2(33.3)$ & $1(16.7)$ & $2(66.7)$ & $1(016.7)$ & $0(0)$ \\
$\quad$ Total & 84 & $\mathbf{7 ( 8 . 3 )}$ & $\mathbf{1 3 ( 1 5 . 5 )}$ & $\mathbf{2 0 ( 2 3 . 8 )} 10(11.9)$ & $\mathbf{6 ( 8 . 3 )}$ & $\mathbf{9 ( 1 2 . 5 )}$ & $\mathbf{1 9}(\mathbf{2 6 . 4})$
\end{tabular}

R0: Not resistant for any antibiotics tested, R1: Resistant to one antibiotic, R2: Resistant to two antibiotics, R3: Resistant to three antibiotics, R4: Resistant to four antibiotics, R5: Resistant to five antibiotics, $\geq$ R6: Resistant to six or more antibiotics, NT: Not Tested, Other isolates: Accinitobacter spp (1), Seratia spp (3) and Proteus spp (2).

Among isolates from treated wastewater, 12/42 (28.6\%) were resistant for six and more antibiotics (e.g. two isolates of Klebsiella spp and one isolates of CoNS were resistant for ten antibiotics, one isolates of Citrobacter spp were also resistant for nine antibiotics), while $8 / 50$ (16\%) were resistant for only one antibiotics and there was no isolates that are not resistant for any of antibiotics tested (Table 6).

Among isolates from both treated and untreated hospital wastewater the overall prevalence of multi-drug resistance (resistant to two and above antibiotics) in this study was found to be 106/134 (79.1\%). Multi-drug resistance in untreated wastewater sample was found to be $64 / 84(76.2 \%)$ whereas in treated wastewater was found to be $42 / 50$ (84\%). In this study isolates from treated wastewater was found to be 1.64 times resistant for many drug than isolates from untreated hospital wastewater (COR:1.64, 95\% CI: 1.15 - 3.29, P: 0.001).

\section{Discussion}

The present study showed that mean heterotrophic plate count $\left(1.6 \times 10^{6}\right.$ $\mathrm{CFU} / \mathrm{mL}$ ), was exceeded the permissible limit of Environment Protection Agency, EPA [16] and Health Protection Agency, HPA [17] $(<1000$ CFU/mL) for treated wastewater. Total coliform count $\left(2.2 \times 10^{6} \mathrm{CFU} / 100 \mathrm{~mL}\right)$ also failed to fulfill the requirements of the revised guidelines on the quality of treated wastewater used in agriculture, in public parks $\left(<5 \times 10^{3} \mathrm{CFU} / 100 \mathrm{~mL}\right)$ [18] [19]. 
Table 6. Multi-drug resistance patterns of bacterial isolates from treated wastewater released from Ayder Referral Hospital, Mekelle, North Ethiopia (September-December 2015).

\begin{tabular}{ccccccccc}
\hline Bacteria isolates & $\begin{array}{c}\text { Number } \\
\text { of isolates }\end{array}$ & $\mathbf{R} 0$ & $\mathbf{R} 1$ & $\mathbf{R} 2$ & $\mathbf{R} 3$ & $\mathbf{R} 4$ & $\mathbf{R} 5$ & $\geq \mathbf{R} 6$ \\
\hline S. aureus & 8 & $0(0)$ & $1(12.5)$ & $2(25)$ & $2(25)$ & $1(12.5)$ & $0(0)$ & $2(25)$ \\
CoNS & 5 & $0(0)$ & $0(0)$ & $0(0)$ & $1(20)$ & $2(40)$ & $1(20)$ & $1(20)$ \\
Klebsiella spp & 10 & $0(0)$ & $1(10)$ & $2(20)$ & $2(20)$ & $1(10)$ & $1(10)$ & $3(30)$ \\
E. coli & 6 & $0(0)$ & $1(16.7)$ & $0(0)$ & $1(16.7)$ & $2(33.3)$ & $0(0)$ & $2(33.3)$ \\
P. aeroginosa & 8 & $0(0)$ & $5(62.5)$ & $1(12.5)$ & $2(25)$ & $\mathrm{NT}$ & $\mathrm{NT}$ & $\mathrm{NT}$ \\
Salmonella spp & 6 & $0(0)$ & $0(0)$ & $1(16.7)$ & $2(33.3)$ & $1(16.7)$ & $0(0)$ & $2(33.3)$ \\
Citrobacter spp & 3 & $0(0)$ & $0(0)$ & $1(33.3)$ & $0(0)$ & $0(0)$ & $0(0)$ & $2(66.7)$ \\
Entrobacter spp & 2 & $0(0)$ & $0(0)$ & $1(50)$ & $0(0)$ & $0(0)$ & $1(50)$ & $0(0)$ \\
Other isolates & 2 & $0(0)$ & $0(0)$ & $0(0)$ & $0(0)$ & $1(50)$ & $1(50)$ & $0(0)$ \\
$\quad$ Total & 50 & $\mathbf{0 ( 0 )}$ & $\mathbf{8 ( 1 6 )}$ & $\mathbf{8 ( 1 6 )}$ & $\mathbf{1 0 ( 2 0 )}$ & $\mathbf{8 ( 1 9 . 0 )}$ & $\mathbf{4}(\mathbf{9 . 5})$ & $\mathbf{1 2 ( 2 8 . 6 )}$ \\
\hline
\end{tabular}

R0: Not resistant for any antibiotics tested, R1: Resistant to one antibiotic, R2: Resistant to two antibiotics, R3: Resistant to three antibiotics, R4: Resistant to four antibiotics, R5: Resistant to five antibiotics, $\geq$ R6: Resistant to six or more antibiotics, NT: Not Tested, Other isolates: Seratia spp (1) and Proteus spp (1).

Microbial contamination of treated hospital wastewater was $2.0 \times 10^{5}$ of fecal coliforms, which was higher than WHO maximum tolerable limit for fecal indicator bacteria $\left(\leq 10^{3} / 100 \mathrm{~mL}\right.$ for unrestricted irrigation and $\leq 10^{5} / 100 \mathrm{~mL}$ for restricted irrigation) [20] [21]. Faecal coliform count was also higher than FAO standard allowable limit of $5000 \mathrm{CFU} / 100 \mathrm{~mL}$ [22]. High density of $E$. coli $(1.1 \times$ $\left.10^{4}\right)$ in treated wastewater was also an indication of fecal pollution of environment due to human activities.

In the present study bacterial isolates such as Klebsiella spp 10 (20\%), P. aeruginosa 8 (16\%), S. aureus 8 (16\%), E. coli 7 (14\%) and salmonella spp $6(12 \%)$ were also frequently detected in treated wastewater. The same study conducted in Ethiopia, Hawassa University Referral Hospital, reported Salmonella spp, Shigella spp, E. coli and $S$. aureus from hospital effluent [3]. Similarly, from Thailand pathogenic bacteria like Vibrio spp and Salmonella spp as well as other potentially pathogenic bacteria were reported [23]. Likewise, study in India showed large numbers of enteric-bacteria, $S$. aureus and $P$. aeruginosa [24]. Study in Tunisia also showed large amount of pathogenic bacteria like Salmonella that have a real impact in human health [25]. The highest prevalence of Salmonella species is due to the fact that it is highly associated with typhoid fever, paratyphoid fever and gastroenteritis, especially of developing countries. Different research also revealed that its survival in fresh water and sewage is less than 60 days and better recovered from sewage system compared to Shigella. The highest microbial contamination of treated wastewater shows wastewater treatment plant may not be efficient with regard to removing bacteria in the final effluent that is discharged to the receiving environment. 
Among bacterial isolates from treated wastewater, $S$. aureus and CoNS were $100 \%$ resistant to penicillin and also E. coli, Klebsiella spp and Citrobacter spp were $100 \%$ resistant to ampicillin. In general most bacterial isolates were highly resistant to tetracycline, doxycycline, cotrimoxazole, amoxicillin-clavulinic acid and ceftriaxone. The same study in India showed simultaneous resistance of isolates for ampicillin, ampicillin with clavulinic acid, cotrimoxazole, tetracycline, first, second and third generation cephalosporins in the final effluent of wastewater treatment plant [26]. Study in Alexandria, Egypt also showed the presence of antibiotic resistant extended spectrum B-lactamase (ESBL) producing bacteria at the end of wastewater purification process [2], posing a risk of its spread to the environment and subsequent human and animal exposure.

Overall resistance of bacterial isolates from THWW for methicillin was found to be 38\%. Methicillin resistant Staphylococci (MRSA and MRCoNS) were not studied well in our country from hospital effluents. But as indicated by Abulreesh [27] from International Conference on biotechnology and environment management, Singapore multidrug resistant staphylococci $(S$. aureus and coagulase negative Staphylococci) had been a common problem and recovered from diverse environmental sources, such as drinking water supplies and hospital environments which warns public health concern since contamination of river and lake with this pathogen may pose risk to the public health associated with Staphylococcal infection and food poisoning.

This study also observed that, most bacterial isolates from the THWW shows higher rate of resistance than bacterial isolates from the UHWW and shows resistant bacteria isolates were able to survive the journey to the inlet of sewage treatment plant and treatment process. The same result was reported from Alice, Eastern Cape province of South Africa [28] and European countries [29] [30], where higher rate of resistance in bacterial isolates from final effluent of wastewater treatment plant was found. Presences of high percentage of drug resistant isolates from the final effluent of WWTP suggest that, hospital wastewater could have contributed massively to the resistances observed among the isolates from the final effluent. These can be due to the fact that, hospital wastewater contains a diverse group of pathogenic, commensal and environmental bacteria. This characteristic composition makes sewage particularly suitable ecological niche for the growth and spread of antibiotic resistance due to selection pressure and horizontal gene transfer [31] [32] [33].

High percentages of multi-drug resistance for the majority of the isolates in THWW were discharged to the environment. This was supported by Study conducted in Switzerland which showed that increased proportions of highly and extremely multi-resistant bacteria among the isolated sulfamethoxazole/ trimethoprim and streptomycin resistant strains in the sample of treated wastewater compared to the wastewater treatment plant inlet sample [34]. Study conducted in Australia [35], Brazil [36] and China [37] that investigates a hospital sewage treatment system also showed, the treatment of the hospital wastewater may not be totally effective in removing multiple drug resistant bacteria and 
resistant gene from hospital wastewater.

The release of MDR bacteria to the environment causes extensive genetic exchange; where opportunistic pathogens (commonly found in free-living communities) may become resistant upon acquiring resistance mechanisms. Therefore, reduction of selective pressure by regulating the use of antibiotics is a key step to undermine the spread of resistance in hospital wastewater in order not to favored resistant strains [38].

\section{Conclusion}

In the present study, high numbers of indicator organisms were obtained from treated hospital wastewater, which exceeded the WHO, HPA, EPA and FAO standard permissible levels. Significant pathogenic and potentially pathogenic bacteria were also isolated from the treated wastewater. There was high prevalence of drug resistant isolates from untreated and treated hospital wastewater suggesting their persistence in the hospital environment, and their ability to pass the processes of treatment plant. Therefore Liquid waste treatment system (Chlorination) should be developed to disinfect pathogens in treated wastewater effluents.

\section{Ethical Approval}

Approved.

\section{Competing Interests}

The authors declare that they have no competing interests.

\section{Author Contributions}

TA participated in its design and performed the laboratory activities. TA analyzed the data and wrote the manuscript. LN, AK and YW reviewed the manuscript. All authors read and approved the final manuscript.

\section{References}

[1] Abdel-Rouf, N., Al-Homaidan, A.A. and Ibraheem, I.B.M. (2012) Microalgae and Wastewater Treatment. Saudi Journal of Biological Sciences, 19, 257-275. https://doi.org/10.1016/j.sjbs.2012.04.005

[2] Amine, A.E.K. (2013) Extended Spectrum Beta-Lactamase Producing Bacteria in Wastewater Alexandria, Egypt. International Journal of Bioscience, Biochemistry, Bioinformatics, 3, 605-608.

[3] Fekadu, S., Merid, Y., Beyene, H., Teshome, W. and Gebre-Selassie, S. (2015) Assessment of Antibiotic and Disinfectant Resistant Bacteria in Hospital Wastewater, South Ethiopia. Journal of Infection in Developing Countries, 9, 149-156. https://doi.org/10.3855/jidc.4808

[4] Beyene, H. and Redaie, G. (2011) Assessment of Waste Stabilization Ponds for the Treatment of Hospital Wastewater: The Case of Hawassa University Referral Hospital. World Applied Sciences Journal, 15, 142-150.

[5] Stalder, T., Barraud, O., Jove, T., Casellas, M., Gaschet, M., Dagot, C., et al. (2014) 
Quantitative and Qualitative Impact of Hospital Effluent on Dissemination of the Integron Pool. The ISME Journal, 8, 768-777.

https://doi.org/10.1038/ismej.2013.189

[6] Keen, P.L. and Patrick, D.M. (2013) Tracking Change: A Look at the Ecological Footprint of Antibiotics and Antimicrobial Resistance. Antibiotics Review, 2, 191-205. https://doi.org/10.3390/antibiotics2020191

[7] Nunez, L. and Moretton, J. (2007) Disinfectant-Resistant Bacteria in Buenos Aires City hospital Wastewater. Brazilian Journal of Microbiology, 38, 644-648. https://doi.org/10.1590/S1517-83822007000400012

[8] Anitha, J. and Jayraaj, I.A. (2012) Isolation and Identification of Bacteria from Biomedical Waste (BMW). International Journal of Pharmacy and Pharmaceutical Sciences, 4, 386-388.

[9] Pandey, A., Afsheen, Ara, F. and Tiwari, S.K. (2011) Isolation and Characterization of Multi Drug Resistance Cultures from Waste Water. Journal of Pharmaceutical and Biomedical Sciences, 13, 1-7.

[10] Diwan, V., Tamhankar, A.J., Khandal, R.K., Shanta, S., Aggarwal, M., Marothi, Y., et al. (2010) Antibiotics and Antibiotic-Resistant Bacteria in Waters Associated with a Hospital in Ujjain, India. BMC Public Health, 10, 414-422. https://doi.org/10.1186/1471-2458-10-414

[11] Pauwels, B. and Verstraete, W. (2006) The Treatment of Hospital Wastewater. An Appraisal. Journal of Water and Health, 4, 405-416.

[12] Tadesse, M.L. and Kumie, A. (2014) Healthcare Waste Generation and Management Practice in Government Health Centers of Addis Ababa, Ethiopia. BMC Public Health, 14, 1221-1239. https://doi.org/10.1186/1471-2458-14-1221

[13] Engdaw, F. (2014) Physioco-Chemical Parameters and Bacteriological Qualities of Water Samples from Wastewater Treatment Pond, University of Gonder, Ethiopia. International Journal of Pharmaceutical and Health Care Research, 2, 192-197.

[14] APHA (2005) Standard Methods for the Examination of Water and Waste Water. American Public Health Association/American Water Works Association/Water Environment Federation $21^{\text {th }}$ ed.

[15] CLSI (2014) Performance Standards for Antimicrobial Susceptibility Testing; Twenty-Fourth Informational Supplement. CLSI Document M100-S24. Clinical and Laboratory Standards Institute, Wayne, PA.

[16] EPA (2002) US Environment Protection Agency, Safe Drinking Water Act Amendments. http://www.epa.Gov/safewater/mcl.Html

[17] Health Protection Agency (HPA) (2005) The Microbiological Examination of Water Samples. National Standard Method QSOP 57, Issue 2. http://www.hpa-standardmethods.org.uk/pdf-sops.asp

[18] Carr, R.M., Blumenthal, U.J. and Mara, D.D. (2004) Guidelines for the Safe Use of Wastewater in Agriculture: Revisiting WHO Guidelines. Water Science \& Technology, 50, 31-38.

[19] Surface Water Quality Standards Notification of the National Environmental Board, No. 8, BE2537 (1994), Issued under the Enhancement and Conservation of National Environmental Quality Act BE2535 (1992), Published in the Royal Government Gazette, Vol. 111, Part 16, Dated February 24, BE2537 (1994).

[20] Ursula, B.J., Mara, D.D., Peasey, A., Ruiz-Palacios, G. and Stott, R. (2000) Guidelines for the Microbiological Quality of Treated Wastewater Used in Agriculture. Bulletin of the World Health Organization, 78, 1104-1116. 
[21] WHO (2006) Guidelines for the Safe Use of Wastewater, Excreta and Greywater, Wastewater Use in Agriculture. World Health Organization, Vol. 2.

[22] FAO (2003) (Regional Office for the Near East) User's Manual for Irrigation with Treated Wastewater. 60 .

[23] Danchaivijitr, S., Wongchanapai, W., Assanasen, S. and Jintanothaitavorn, D. (2005) Microbial and Heavy Metal Contamination of Treated Hospital Wastewater in Thailand. Journal of the Medical Association of Thailand, 88, 59-64.

[24] Chitnis, V., Chitnis, D., Patil, S. and Kant, R. (2000) Hospital Effluent: A Source of Multiple Drug-Resistant Bacteria. Current Science, 79, 989-991.

[25] Salem, I.B., Ouardani, I., Hassine and MAouni, M. (2011) Bacteriological and Physico-Chemical Assessment of Wastewater in Different Region of Tunisia: Impact on Human Health. BMC Research Notes, 4, 144-155. https://doi.org/10.1186/1756-0500-4-144

[26] Katouli, M., Thompson, J.M., Gundogdu, A. and Stratton, H.M. (2012) Antibiotic Resistant Bacteria in Hospital Wastewaters and Sewage Treatment Plant. Science Forum and Stakeholder Engagement: Building Linkages, Collaboration and Science Quality, 225-229.

[27] Abulreesh, H.H. (2011) Multidrug-Resistant Staphylococci in the Environment. In: International Conference on Biotechnology and Environment Management, Vol. 18, IACSIT Press, Singapore.

[28] Iweriebor, B.C., Gaqavu, S., Chikwelu Obi, L., Nwodo, U.U. and Okoh, A. (2015) Antibiotic Susceptibilities of Enterococcus Species Isolated from Hospital and Domestic Wastewater Effluents in Alice, Eastern Cape Province of South Africa. International Journal of Environmental Research and Public Health 12, 4231-4246. https://doi.org/10.3390/ijerph120404231

[29] Blanch, A.R., Caplin, J.L., IversenAKu, H.I., Taylor, M.A. and Vilanova, X. (2003) Comparison of Enterococcal Populations Related to Urban and Hospital Wastewater in Various Climatic and Geographic European Regions. Journal of Applied Microbiology, 94, 994-1002. https://doi.org/10.1046/j.1365-2672.2003.01919.x

[30] Servais, P. and Passerat, J. (2009) Antimicrobial Resistance of Fecal Bacteria in Waters of Seine River Watershed (France). Science of the Total Environment, 8, 365-372. https://doi.org/10.1016/j.scitotenv.2009.09.042

[31] Periasamy, D. and Sundaram, A. (2013) A Novel Approach for Pathogen Reduction in Wastewater Treatment. Journal of Environmental Health Science and Engineering, 11, 12-21. https://doi.org/10.1186/2052-336X-11-12

[32] Cantona, R., Horcajadad, J.P., Oliverb, A., Garbajosaa, P.R. and Vilab, J. (2013) Inappropriate Use of Antibiotics in Hospitals: The Complex Relationship between Antibiotic Use and Antimicrobial Resistance. Enfermedades Infecciosas y Microbiología Clínica, 31, 3-11. https://doi.org/10.1016/S0213-005X(13)70126-5

[33] Davies, J. and Davies, D. (2010) Origins and Evolution of Antibiotic Resistance. Microbiology and Molecular Biology Reviews, 74, 417-433. https://doi.org/10.1128/MMBR.00016-10

[34] Czekalski, N., Berthold, T., Caucci, S., Egli, A. and Burgmann, H. (2012) Increased Levels of Multi-Resistant Bacteria and Resistance Gene after Wastewater Treatment and Their Dissimination into Lake Geneva, Switzerland. Frontiers in Microbiology, 3, 1-11.

[35] Omar Faruk, S.M., Masudul Azad, C.A.M. and Nayeem, U.K. (2014) Isolation of Cefixime Resistant Salmonella from Hospitals Waste and Profiling Multi-Drug Resistance Pattern of the Selected Isolates. International Research Journal of Biological Sciences, 3, 86-92. 
[36] Chagas, T.P., Seki, L.M., Cury, J.C., Oliveira, J.A., Dávila, A.M., Silva, D.M., et al. (2011) Multiresistance, Beta-Lactamase-Encoding Genes and Bacterial Diversity in Hospital Wastewater in Rio de Janeiro Brazil. Journal of Applied Microbiology, 111, 572-581. https://doi.org/10.1111/j.1365-2672.2011.05072.x

[37] Yuan, Q.B., Guo, M.-T. and Yang, J. (2015) Fate of Antibiotic Resistant Bacteria and Genes during Wastewater Chlorination: Implication for Antibiotic Resistance Control. PLoS ONE, 10, e0119403. https://doi.org/10.1371/journal.pone.0119403

[38] Bush, K., Courvalin, P., Dantas, G., Davies, J., Eisenstein, B., et al. (2011) Tackling Antibiotic Resistance. Nature Reviews Microbiology, 9, 894-896.

https://doi.org/10.1038/nrmicro2693 